\title{
Aberrant iPSC-derived human astrocytes in Alzheimer's disease
}

\author{
VC Jones ${ }^{1}$, R Atkinson-Dell ${ }^{2}$, A Verkhratsky ${ }^{2,3}$ and L Mohamet $^{\star, 2}$
}

The pathological potential of human astroglia in Alzheimer's disease (AD) was analysed in vitro using induced pluripotent stem cell (iPSC) technology. Here, we report development of a human iPSC-derived astrocyte model created from healthy individuals and patients with either early-onset familial $A D$ (FAD) or the late-onset sporadic form of $A D$ (SAD). Our chemically defined and highly efficient model provides $>95 \%$ homogeneous populations of human astrocytes within 30 days of differentiation from cortical neural progenitor cells (NPCs). All astrocytes expressed functional markers including glial fibrillary acidic protein (GFAP), excitatory amino acid transporter-1 (EAAT1), S100B and glutamine synthetase (GS) comparable to that of adult astrocytes in vivo. However, induced astrocytes derived from both SAD and FAD patients exhibit a pronounced pathological phenotype, with a significantly less complex morphological appearance, overall atrophic profiles and abnormal localisation of key functional astroglial markers. Furthermore, NPCs derived from identical patients did not show any differences, therefore, validating that remodelled astroglia are not as a result of defective neural intermediates. This work not only presents a novel model to study the mechanisms of human astrocytes in vitro, but also provides an ideal platform for further interrogation of early astroglial cell autonomous events in $A D$ and the possibility of identification of novel therapeutic targets for the treatment of $A D$.

Cell Death and Disease (2017) 8, e2696; doi:10.1038/cddis.2017.89; published online 23 March 2017

All diseases, including neurological disorders, can be broadly defined as homeostatic failures within tissues, organs or systems. Astroglia are highly heterogeneous neural cells primarily responsible for homeostasis and neuroprotection in the CNS. Astrocytes are fundamental for synaptogenesis and synaptic maintenance; they control ion homeostasis in the CNS interstitium and are indispensable for turnover of major neurotransmitters, such as glutamate, GABA and adenosine. ${ }^{1,2}$ In addition, astroglial cells are endowed with an evolutionary conserved defensive programme known as reactive gliosis, which develops in response to CNS lesions and is manifested by a spectrum of disease-specific cellular responses including hypertrophy and upregulation of intermediate filaments. ${ }^{3,4}$ Astrocytopathy is a central element of neurological disorders and, depending on the disease context, astrocytes undergo complex changes, which vary from astroglial atrophy with loss of function, to pathological remodelling or reactivity and may develop alone or in combination. ${ }^{5-8}$ In Alzheimer's disease (AD) animal models, atrophic astrocytes have been detected at the earliest stages of the disease, with hypertrophic reactive astrocytes appearing later in disease progression, in response to their proximity to extracellular accumulations of $\beta$-amyloid $(\mathrm{A} \beta){ }^{9-12}$ Similarly, signs of astroglial reactivity and atrophy were detected in $A D$ patients by positron emission tomography using ${ }^{11} \mathrm{C}$-deteriumL-deprenyl. ${ }^{13}$ These observations led to a hypothesis of glial paralysis being a fundamental factor in evolution of $A D .^{14}$

The sporadic (late-onset) form of $A D(S A D)$, which is without significant non-Mendelian genetic bias, dominates human pathology. Nevertheless, most of our knowledge of AD derives from studies that utilise cell- and animal-based models of the clinically rare, early-onset, dominantly inherited familial $A D$ (FAD). The cellular pathology of astroglia in the context of human $A D$ remains enigmatic, mainly because of severe limitations of animal models, which, although reproducing some pathological features of the disease, do not mimic its progression in full. Neuronal loss and cognitive deficits, which are the hallmarks of $A D$ in humans, are limited in animal models ${ }^{15}$ and there is an increasing body of evidence showing that significant differences exist between rodent and human astrocytes. ${ }^{16}$ Although induced pluripotent stem cell (iPSC) technology can be used to investigate human astrocyte development and function, only a limited number of studies have described homogeneous generation of healthy astrocytes. ${ }^{17-22}$ Previous work has shown that neurones differentiated from SAD- and FAD-iPSCs resemble pathologically affected cells in vivo and express key disease hallmarks; ${ }^{23-25}$ however, there is still a pressing need for tools to provide robust, homogeneous astrocyte populations to reveal astroglial contribution to neurodegenerative diseases. Here we report the generation of enriched mature astroglia from human iPSCs derived from patients with confirmed FAD and $S A D$ as well as from a healthy control. We found that, although astrocytes derived from $A D$ patients express the same canonical markers of mature healthy astrocytes, their morphological appearance and cellular phenotype is significantly distorted. Notably, we did not observe any differences in early neuronal commitment in early $\mathrm{AD}$-derived neurones

\footnotetext{
${ }^{1}$ The University of Central Lancashire, Preston PR1 2HE, UK; ${ }^{2}$ The University of Manchester, Manchester M13 9PT, UK and ${ }^{3}$ Achucarro Center for Neuroscience, IKERBASQUE, Basque Foundation for Science, Bilbao 48011, Spain

${ }^{*}$ Corresponding author: L Mohamet, Faculty of Biology, Medicine and Health, The University of Manchester, AV Hill Building, Manchester M13 9PT, UK. Tel: +44 (0) 161 2756724; Fax: +0161 2756734; E-mail:lisa.mohamet@manchester.ac.uk

Received 22.12.16; revised 02.2.17; accepted 06.2.17; Edited by G Raschella'
} 
compared with healthy controls, indicating the cell autonomous pathological potential of astroglia and further confirming that cellular pathology does not stem from defective neural progenitors.

\section{Results}

Generation of neural cell lineages. Cortical NPCs were propagated in monolayer culture and maintained as described in ref. 24. Both FAD and SAD NPCs showed indistinguishable culture morphology to healthy NPCs $(N=5$ per cell line; Figures $1 \mathrm{a}-\mathrm{c}$ ). Although a degree of variability in cell proliferation and viability was observed between cells from each individual, no significant differences in NPC growth rates were identified ( $N=4$ per cell line, two-way KruskalWallis, Figures 1d and e). Phenotypic identification of NPCs showed positive expression of the transcription factors SOX1 and PAX6, and the filamentous marker, nestin (Figures 1f-n). However, no significant difference in NPC marker expression was observed irrespective of genotype (quantified by the proportion of immunoreactive nestin + cells; $N=4$ per cell line, analysis of variance (ANOVA), $\mathrm{F}_{(2,9)}=0.022, \quad P=\mathrm{NS}$, Figure 10). Furthermore, under terminal neuronal differentiation conditions for 35-40 days, all patient samples showed positive expression of the neural marker $\beta$-III-tubulin (Figures 1p-r). No significant difference was detected in the efficiency of early neuronal induction (as measured by the proportion of $\beta$-III-tubulin+ neurones) between any individual $\left(N=4\right.$ per cell line, ANOVA, $F_{(2,9)}=0.128, P=N S$, Figure $\left.1 \mathrm{~s}\right)$. Expression of the mature deep-layer cortical neuronal marker, CTIP2 was observed throughout the cultures from each patient, indicating maintenance of cortical identity following 40 days in culture (Figures 1t-v). In a separate study, we also show that cortical neurones derived from identical FAD and SAD patients exhibit elevated pathological $A \beta-42$ secretion and hyperphosphorylated tau species compared with healthy individuals, therefore validating an AD phenotype in these cells (data not shown). The results presented herein reveal that both SAD and FAD NPCs retain their characteristic morphology, expression of canonical markers and are able to generate mature cortical neurones with the same efficiency as healthy derived NPCs, Importantly, therefore, any changes in astroglia are not as a result of defective neuronal intermediates.

To independently evaluate astroglial commitment, we adapted a defined differentiation protocol $^{18}$ to generate purified astroglial cultures. We included additional culture supplementation of EGF and insulin, which have been shown to increase glial commitment, primary astrocyte proliferation and sub-culturing in vitro. ${ }^{26,27}$ Astrocytes were generated within 30 days following induction from cortical NPCs in each cell line as confirmed by positive immunoreactivity to the canonical marker, GFAP and mature astrocyte markers; EAAT1, S100B and GS (Figures 2a-d). Quantification of GFAP, EAAT1, S100B and GS immunoreactive cells revealed near-pure expression in all astrocytes and no significant differences were observed between astrocytes derived from healthy control, FAD and SAD samples ( $N=5$ differentiations per cell line (minimum 120 cells per group), ANOVA,
Supplementary Figures S1A-D). We also examined astrocyte proliferation and viability (day 14 following induction) in both $\mathrm{AD}$ and control cells. Despite some variability in growth rates from each individual, no significant differences were observed in overall astrocyte proliferation or viability $(N=3$ per cell line, two-way Kruskal-Wallis, Supplementary Figures S1E and F). These results demonstrate that both PSEN1 mutant and $\mathrm{ApoE}^{+/+}$NPCs are capable of generating astroglia in vitro and retain expression of markers consistent with mature astrocytes in vivo.

\section{Altered cellular heterogeneity in FAD and SAD astro-} cytes. Our initial observations revealed considerable differences in astrocyte morphology in cells derived from ADNPCs compared with healthy controls. In healthy astrocytes, GFAP staining intensities did not differ from cell to cell, but overall morphology of individual cells across the population varied significantly (Kruskal-Wallis; $\mathrm{H}_{(2)}=13.161, P=0.001$, $N=3$ differentiations). The majority of healthy astrocytes (Figure 2g; 61.2\% $\pm 6.64 ; 93 / 145$ cells) displayed long (defined as $>1 \times$ cell body width) branching fine processes consistent with archetypal astrocyte phenotype (herein referred to as 'arborised'; Figure 2e). A significantly smaller population showed a process-devoid, fibroblast-like phenotype ('fibroblast-like'; 24.9\% \pm 8.45 ; 33/145 cells; DunnBonferroni versus arborised cells, $P=0.002$; Figure 2f, arrow). The remaining cells were thin, highly polarised, but essentially process-devoid cells ('polarised'; $13.9 \% \pm 3.59$; 19/145 cells; Dunn-Bonferroni versus arborised cells, $P=0.017$; versus process-devoid cells, NS; Figure 2f, arrowhead). In contrast, FAD and SAD astrocytes displayed reduced morphological heterogeneity compared with healthy cells. Specifically, FAD astrocytes comprise a significantly greater proportion of fibroblast-like cells than any other morphological type (Figure 3a, quantified in Figure 3e; fibroblast-like cells, $97.0 \% \pm 1.38 ; 150 / 156$ cells; KruskalWallis, $H_{(2)}=10.091, P=0.006$; Dunn-Bonferroni versus arborised cells, $P=0.047$; versus polarised cells, $P=0.008$; $N=3$ differentiations), despite their expression of mature astrocyte markers. This proportion of fibroblast-like cells was significantly greater than that seen with healthy astrocytes (Figure 5a; $\mathrm{H}_{(2)}=14.530, \quad P=0.001$; Dunn-Bonferroni, $P=0.008)$. FAD astrocytes with an archetypal astrocyte-like arborised morphology were rare and their relative proportion was significantly reduced in comparison with healthy control cells $(1.94 \% \pm 0.84 ; \quad 4 / 156 \quad$ cells; Kruskal-Wallis, $\mathrm{H}_{(2)}=14.839, P=0.001$; Dunn-Bonferroni, $P=0.011$ ). Similarly, SAD astrocyte cultures comprise mainly process-devoid fibroblast-like cells (Figure 4a; quantified in Figure 4e; $96.4 \% \pm 2.64 ; 126 / 130$ cells) with significantly fewer arborised cells $(2.72 \% \pm 2.72 ; 3 / 130$ cells; Kruskal-Wallis, $\mathrm{H}_{(2)}=11.129, P=0.004$; Dunn-Bonferroni versus fibroblastlike, $P=0.013 ; N=3$ differentiations) or polarised cells $(0.87 \% \pm 0.87 ; 1 / 130$ cells; Dunn-Bonferroni versus fibroblast-like, $P=0.010)$. Again, there was a significant increase in the proportion of fibroblast-like cells in SAD astrocytes compared with healthy astrocytes (Figure 4a; Dunn-Bonferroni, $P=0.004$ ) concomitant with a reduction in the proportion of arborised cells (Dunn-Bonferroni, $P=0.002$ ). Notably, there was no significant difference in the relative proportions 
Healthy
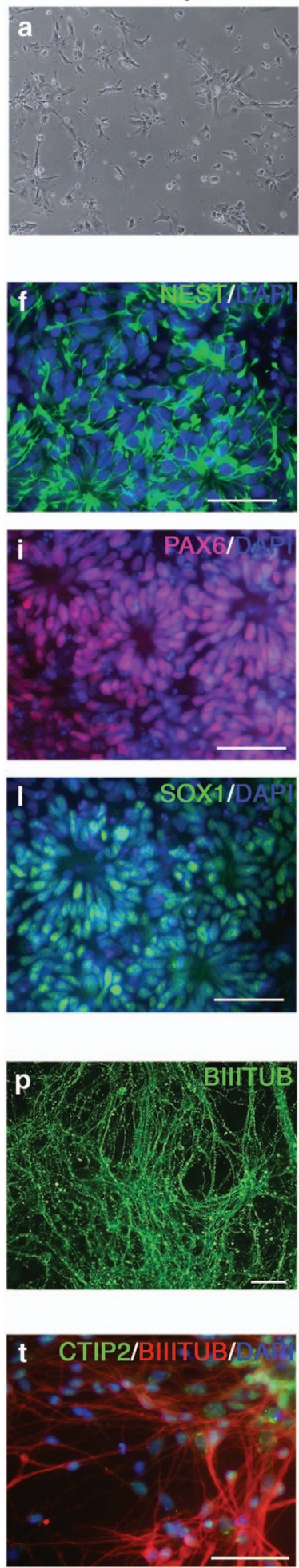

FAD
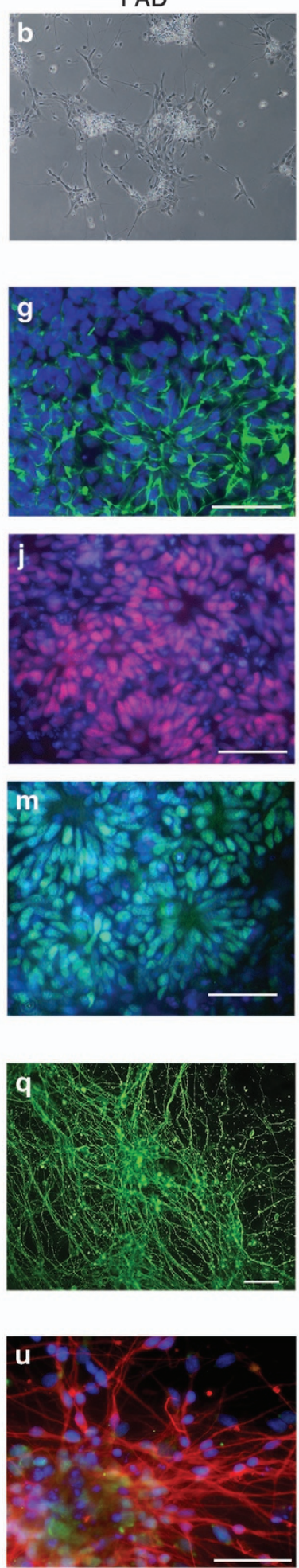

SAD
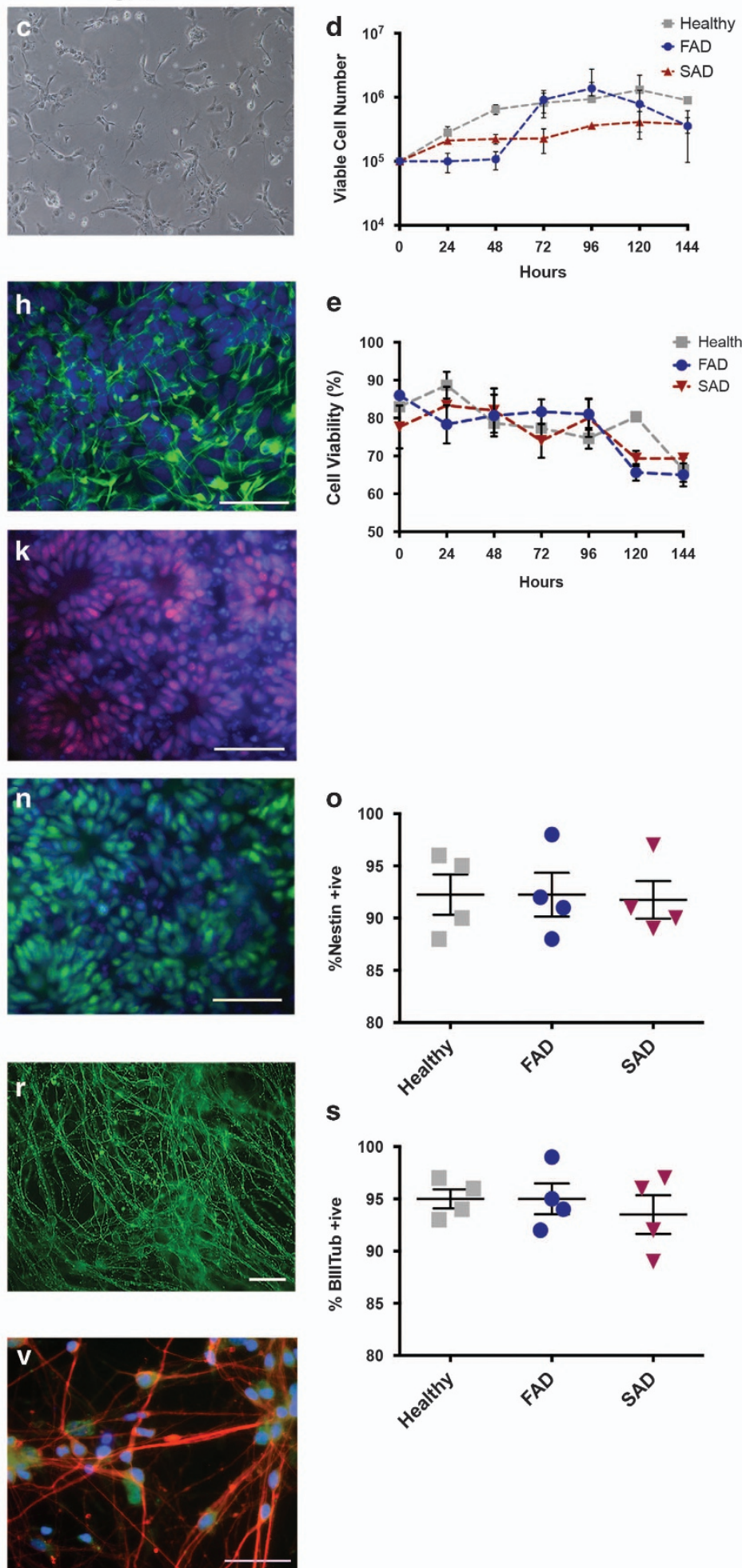

e

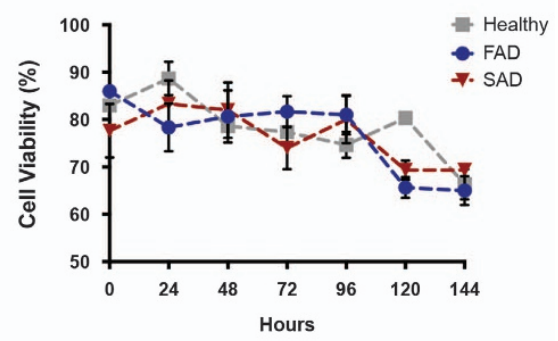

S
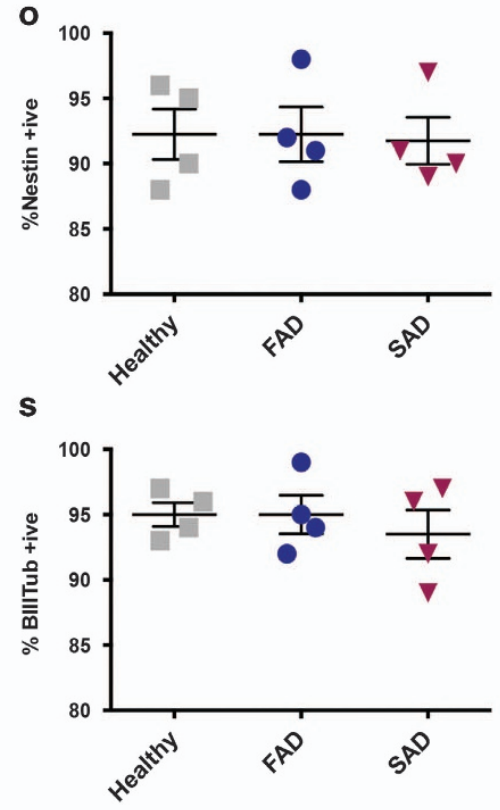

Figure 1 Directed differentiation of healthy and AD-neural progenitor cells into cortical neurones. (a-c) NPCs were seeded at $1 \times 10^{5}$ per well and propagated in monolayer culture for 6/7 days. FAD and SAD cortical NPCs showed indistinguishable culture morphology with healthy (control) NPCs ( $N=5$ per cell line). (d and e) No significant differences in NPC growth rates were identified ( $N=4$ per cell line, two-way Kruskal-Wallis $P=N S)$. ( $f-n)$ IPS-derived NPCs from healthy (control), FAD and SAD patients were assessed for canonical marker expression. Progenitor cells formed polarised rosettes expressing nestin (green; $\mathbf{f - h}$ ), PAX6 (red; I-k) and SOX1 (green, I-n). (o) No significant difference in nestin + cells was observed between healthy and AD cell lines ( $N=4$ per cell line, ANOVA, $\left.\mathrm{F}_{(2,9)}=0.022, P=N S\right)$. ( $\mathrm{p}-\mathrm{r}$ ) Under terminal neuronal differentiation conditions for 35-40 days, all patient samples showed positive expression of the neural marker $\beta$-III-tubulin (green). (s) No significant difference in the proportion of $\beta$-III-tubulin+ neurones between any individual ( $N=4$ per group, ANOVA, $\left.\mathrm{F}_{(2,9)}=0.128, P=\mathrm{NS}\right)$. ( $\left.\mathrm{t}-\mathrm{v}\right)$ Expression of the mature deep-layer cortical neuronal marker, CTIP2 was observed throughout cultures from each patient (green). Scale bars, $50 \mu \mathrm{m}$ 

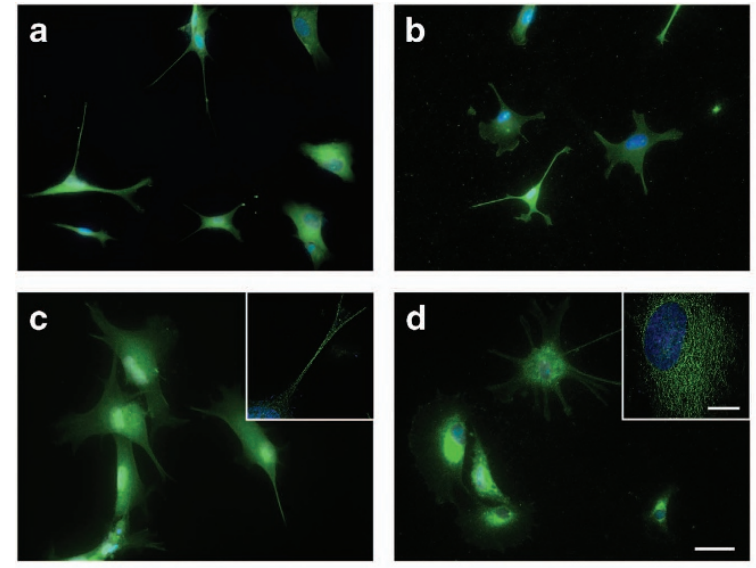

g

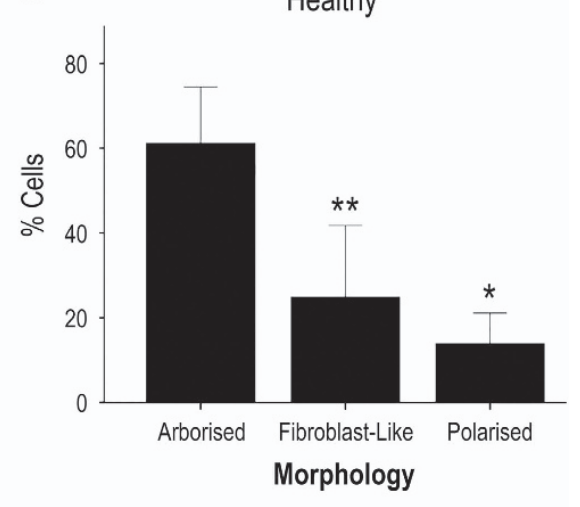

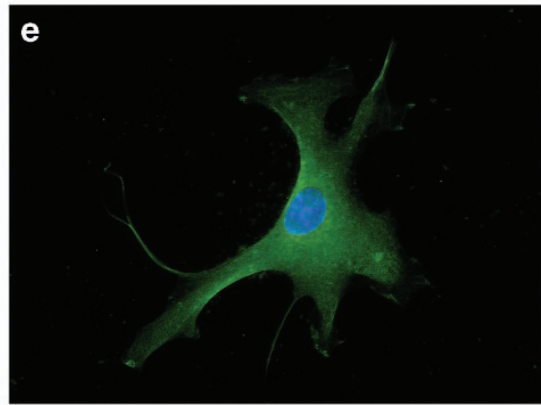

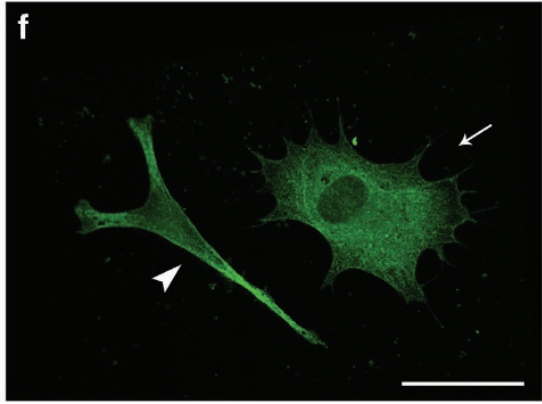

Figure 2 Induced astrocytes derived from healthy patient fibroblasts express mature astrocyte markers and exhibit varied morphologies. Induced astrocytes were generated in 30 days and confirmed by positive immunoreactivity to the functional markers: GFAP (a), S100B (b), EAAT1 (c) and GS (d). Immunostaining is shown in green, DAPI counterstained nuclei are shown in blue. Heterogeneity of morphology within the population of induced astrocytes was evident, with cells falling into three broad categories when stained for GFAP: arborised cells (e); polarised cells (f, arrowhead); or fibroblast-like, process-devoid cells (f, arrow); summarised in $(\mathbf{g})$. ${ }^{* *} P<0.005,{ }^{*} P<0.05$. Scale bars, $50 \mu \mathrm{m}$ (inset, $20 \mu \mathrm{m}$ )
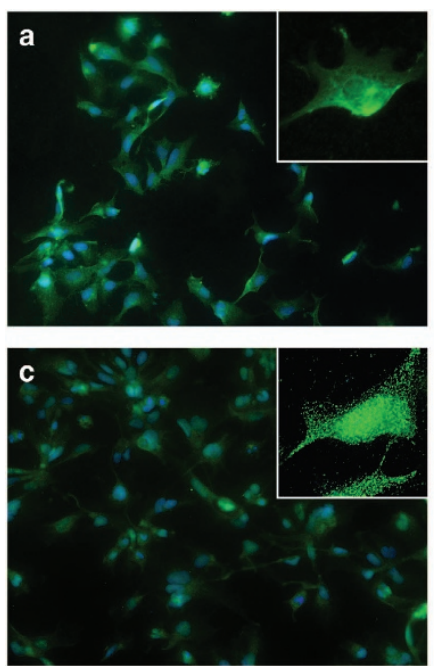
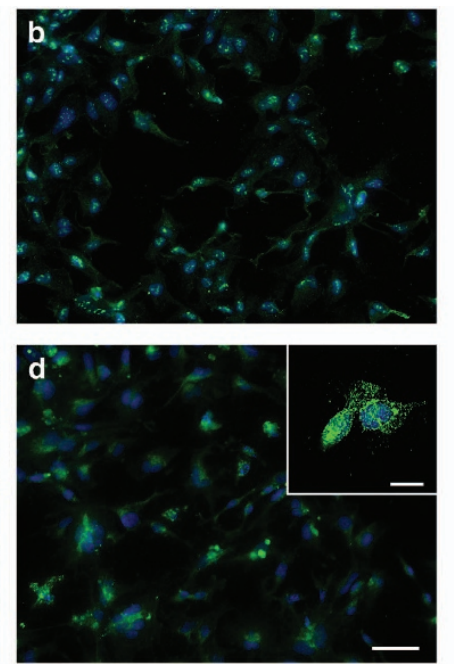

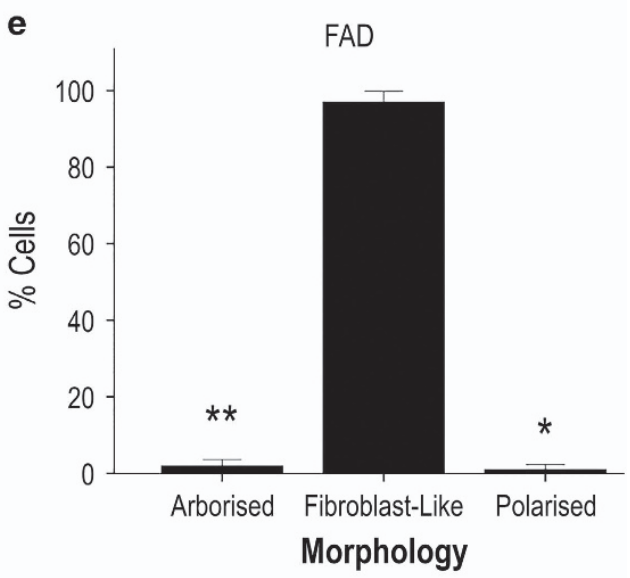

Figure 3 Induced astrocytes derived from PSEN1 M146L FAD patient fibroblasts express normal astrocyte markers but show reduced morphological heterogeneity compared with healthy cells. Induced astrocytes were immunostained for GFAP (a), S100B (b), EAAT1 (c) and GS (d). Immunostaining is shown in green, DAPI counterstained nuclei are shown in blue; $>95 \%$ of observed cells were positive for all markers tested. The significant majority of FAD astrocytes display a fibroblast-like, process-devoid appearance (summarised in $\mathbf{e}$ ). ${ }^{\star *} P<0.005,{ }^{*} P<0.05$. Scale bars, $50 \mu \mathrm{m}$ (inset, $20 \mu \mathrm{m}$ )

of each morphological cell type between the FAD and SAD astrocyte groups. Moreover, no significant difference in immunoreactive GFAP intensity was seen in any group (Kruskal-Wallis, $\mathrm{H}_{(2)}=4.496, P=\mathrm{NS}$; Figure $5 f$ ), nor was any loss of nuclear integrity or fragmentation of the GFAP cytoskeleton observed. Taken together, these data demonstrate that astrocytes derived from patients carrying a PSEN1 M146L mutation or both ApoE4 alleles display a significant 

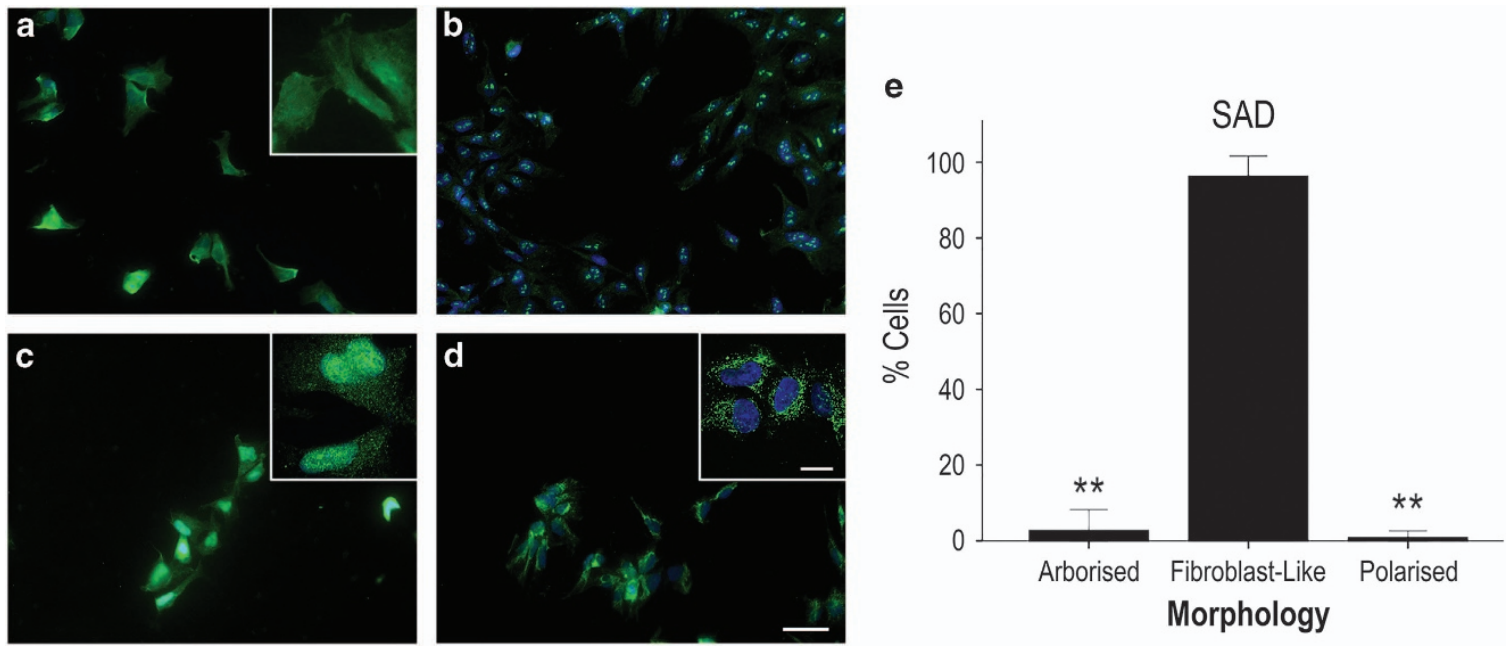

Figure 4 Induced astrocytes derived from $A p o E 4^{+/+}$SAD patient fibroblasts express classical astrocyte markers but show reduced morphological heterogeneity compared with healthy cells. Induced astrocytes were immunostained for GFAP (a), S100B (b), EAAT1 (c) and GS (d). Immunostaining is shown in green, DAPI counterstained nuclei are shown in blue. All observed cells were positive for all markers. A significant majority of SAD astrocytes displayed a fibroblast-like appearance, indicating limited heterogeneity of morphology compared with healthy controls; summarised in (e). ${ }^{\star \star} P<0.005$. Scale bars, $50 \mu \mathrm{m}$ (inset, $20 \mu \mathrm{m}$ )

reduction in morphological heterogeneity when compared with those derived from healthy individuals.

Induced astrocytes from FAD and SAD patients display cellular atrophy. Three-dimensional reconstruction of the entire GFAP cytoskeleton of astrocytes (Figure $6 \mathrm{~b}$ top and Supplementary Movie S1) permitted comparisons of surface area and volume (Figures $5 c$ and $d$ ). We found significant differences between healthy, FAD and SAD astrocytes in both their surface area and volume (surface area, Kruskal-Wallis; $\mathrm{H}_{(2)}=48.454, P<0.001$; volume, Kruskal-Wallis; $\mathrm{H}_{(2)}=48.085$, $P<0.001)$. Specifically, FAD astrocytes displayed a significantly reduced GFAP surface area and volume $\left(2220.4 \pm 204.6 \mu \mathrm{m}^{2}\right.$ and $2574.4 \pm 307.5 \mu \mathrm{m}^{3}$, respectively; $N=3$ per group) in comparison with healthy astrocytes $\left(9978.0 \pm 1048.0 \mu \mathrm{m}^{2}\right.$ and $20867.7 \pm 2102.2 \mu \mathrm{m}^{3}$, respectively; $N=3$; Dunn-Bonferroni pairwise comparisons: surface area, $P<0.001$; volume, $P<0.001)$. There was also a significant reduction in GFAP surface area and volume in SAD astrocytes (1853.5 \pm $171.0 \mu \mathrm{m}^{2}$ and $2301.7 \pm 270.2 \mu \mathrm{m}^{3}$, respectively; $N=3$ per group) when compared with healthy astrocytes (Dunn-Bonferroni pairwise comparisons: surface area, $P<0.001$; volume, $P<0.001)$. No significant difference was observed in either the surface area or volume between FAD and SAD astrocytes (Figures 4c and d), suggesting that astrocytic atrophy may be a common feature in both forms of the disease.

To quantify astrocyte arborisation, we calculated the surface area to volume ratio, SA:Vol (Figure 5e). A larger SA:Vol ratio would indicate a cell with a relatively small cell body (low volume) displaying many processes (large surface area), whereas a small SA:Vol ratio would indicate a more rounded, fibroblast-like cell (large volume) lacking processes (low surface area). We found that the SA:Vol ratio was significantly higher in healthy astrocytes $\left(2.12 \pm 0.07 \mu \mathrm{m}^{-1}\right)$ compared with either FAD or SAD cells $\left(1.14 \pm 0.05 \mu \mathrm{m}^{-1}\right.$ and $1.19 \pm 0.05 \mu \mathrm{m}^{-1}$, respectively; Kruskal-Wallis; $\mathrm{H}_{(2)}=47.385$,
$P<0.001$, Dunn-Bonferroni pairwise comparisons: healthy versus FAD and SAD, both $P<0.001$; FAD versus $S A D$, $P=\mathrm{NS}$ ), indicating a greater degree of arborisation in healthy over diseased cells. Again, these data reveal very little difference between FAD and SAD astrocytes, indicating that aberrant astrocyte morphology is a shared feature of both forms of the disease.

Aberrant expression and localisation of S100B, EAAT1 and GS in AD astrocytes. In healthy astrocytes, S100B expression was observed throughout the cytoplasm and, to a lesser extent, in the nucleus, consistent with normal astrocytes in vivo (Figure 6a, top panel; Figure 6b, left; Supplementary Movie S1). In contrast, both FAD and SAD astrocytes displayed multiple large accumulations of $\mathrm{S100B}$ localised to the nucleus, with very little staining in other cellular compartments (Figure 6a, middle and bottom panels, respectively). To confirm that $\mathrm{S} 100 \mathrm{~B}$ was confined within the nuclei of diseased cells, we utilised z-sectioning confocal microscopy and IsoSurface reconstruction to render 3D models of the staining. Renders of GFAP and DAPI staining were also made to permit comparisons. In both FAD and SAD astrocytes, S100B expression was restricted to the nucleus and confined to multiple, discrete foci following a similar pattern to that of nucleoli (Figure 6b, centre and right; Supplementary Movies S2 and S3, respectively). Fluorescence intensity analysis indicated that $\mathrm{S100B}$ levels were significantly reduced in both FAD and SAD compared with controls, but unchanged relative to each other (ANOVA, $F_{(2,16)}=44.509, P<0.001$; Hochberg's GT2, healthy versus FAD and SAD $P<0.001$, FAD versus SAD $P=N S$; Figure $5 \mathrm{~g}$ ). In both healthy and FAD astrocytes, EAAT1 revealed a uniformed pattern of fluorescence extended throughout the cell (Figures $2 \mathrm{c}$ and $3 \mathrm{c}$, respectively). In contrast, SAD astrocytes exhibited confined nuclear accumulation of EAAT1 (Figure 4c). Quantification of fluorescence 


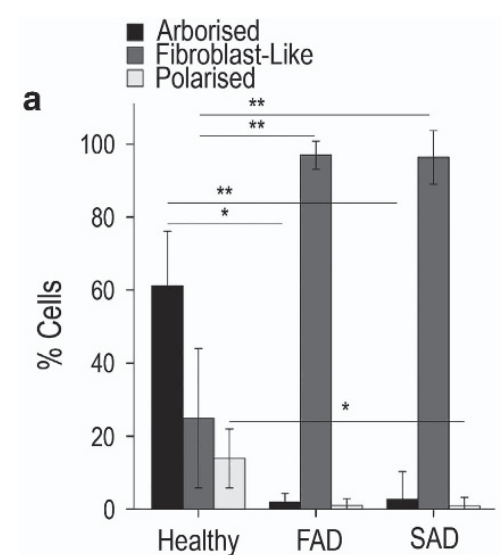

C

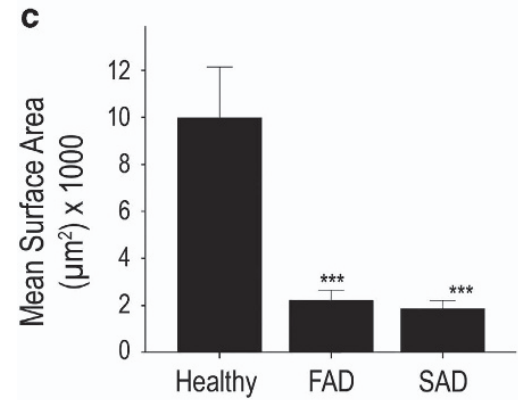

b
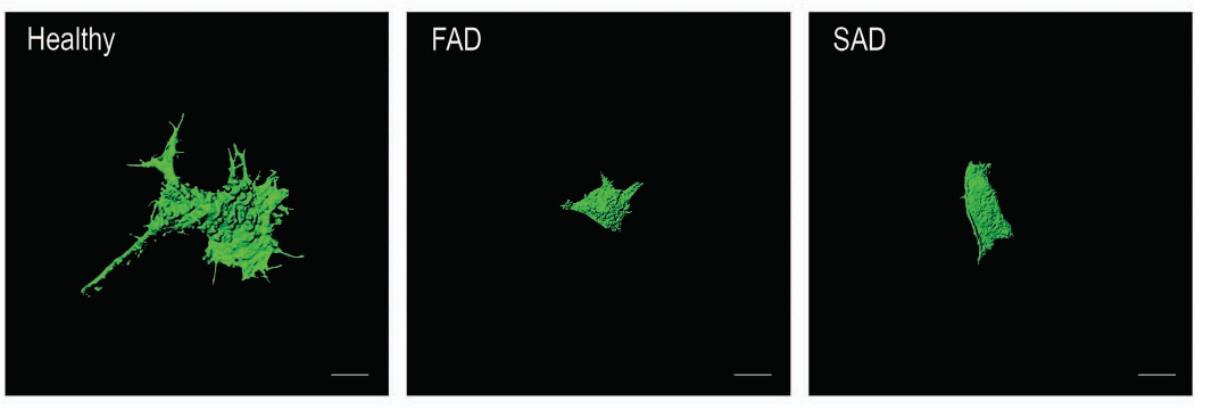

d

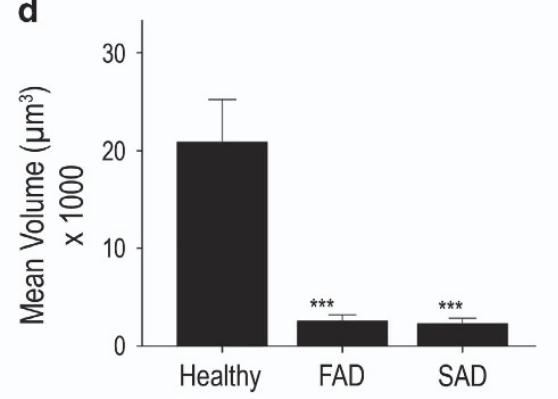

e

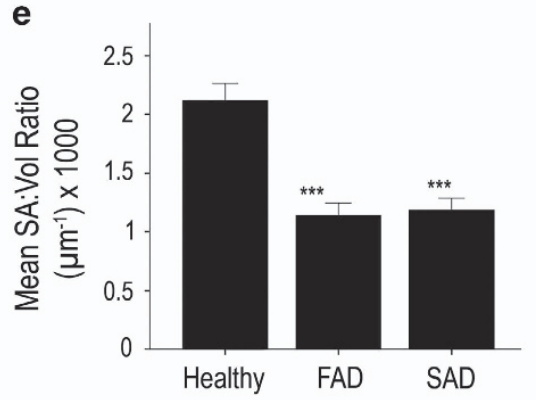

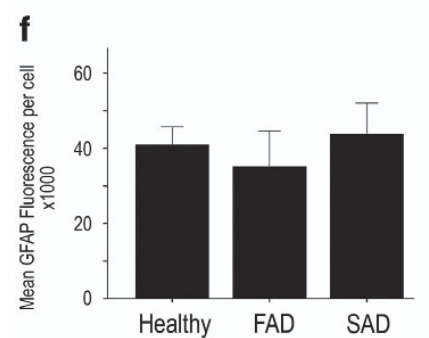
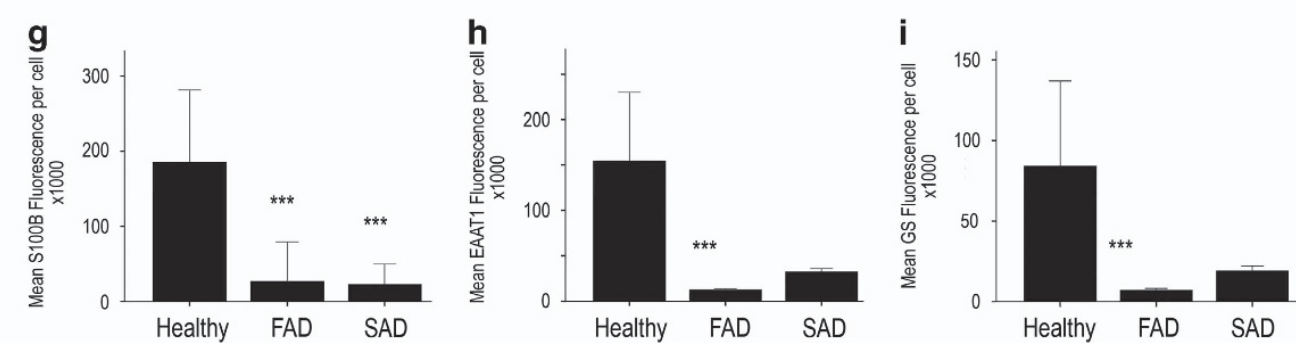

Figure 5 Astrocytes derived from PSEN1 M146L FAD and ApoE4 ${ }^{+/+}$SAD patients exhibit significant atrophy when compared with those from healthy patients as revealed by visual binning according to overall morphology (a). Exemplar 3D IsoSurface renders constructed from serial confocal z-stacks display clear differences in cell size and overall morphology (b). Scale bar, $10 \mu \mathrm{m}$. Quantification of cells using these renders by way of surface area (c), cell volume (d) and SA:Vol ratio (e) reveal significant differences in all aspects of cellular morphology between healthy and diseased astrocytes. Quantification of mean fluorescence intensity per immunoreactive cell reveals no significant difference in GFAP staining intensities between AD and control astrocytes (f) but S100B, EAAT1 and GS intensities are reduced in both FAD and $S A D$ cells $\left(\mathbf{g}-\mathbf{i}\right.$, respectively). ${ }^{* *} P<0.001$, ${ }^{* *} P<0.005,{ }^{*} P<0.05$

intensity revealed that EAAT1 levels were lower in both FAD and SAD astrocytes compared with healthy cells, although only FAD differed significantly (Kruskal-Wallis; $\mathrm{H}_{(2)}=4.235$, $P=0.001$; Bonferroni, healthy versus $\mathrm{FAD}, P<0.001$, versus $\mathrm{SAD}, P=\mathrm{NS}$; Figure $5 \mathrm{~h}$ ). GS immunoreactivity in healthy astrocytes revealed a uniform pattern of fine puncta throughout the cytoplasm (Figure 2d, inset). In diseased cells, however, this smooth pattern was lost and replaced with a distinctly more clumped pattern of staining with much larger puncta (FAD, Figure 3d, inset; SAD, Figure 4d, inset). Fluorescence intensity analysis revealed GS levels were lower in both FAD and SAD astrocytes compared with healthy cells, although only FAD differed significantly (Kruskal-Wallis; $\mathrm{H}_{(2)}=13.430, P=0.001$; Bonferroni, healthy versus $\mathrm{FAD}$, $P=0.001$, versus SAD, $P=\mathrm{NS}$; Figure 5i). Overall these data reveal a drastic reorganisation of key proteins associated with astrocyte function in both FAD and SAD.
Altered non-stimulated release of soluble inflammatory mediators in AD Astrocytes. Non-stimulated astrocytes were cultured under adherent culture conditions and astrocyte conditioned medium (ACM) simultaneously probed for 36 soluble mediators (Supplementary Table S1) using a proteomic array (Supplementary Figures S2A-C). Astrocytes, irrespective of their genotype, constitutively secreted only three cytokines: IL-8 (CXCL8), MCP-1 (CCL2) and TIMP-2. However, SAD astrocytes additionally secreted RANTES (CCL5) and MIP-1 $\beta$. Quantification of expression levels using densitometry showed that IL-8 and MCP-1 secretion was significantly decreased in both non-stimulated ACM from FAD- and SAD-derived astrocytes compared with control ACM (Dunn-Bonferroni; $P<0.001$ for both, $N=3$; Supplementary Figure S2D). These results show that human astrocytes secrete a distinct set of soluble mediators under non-stimulated physiological conditions 


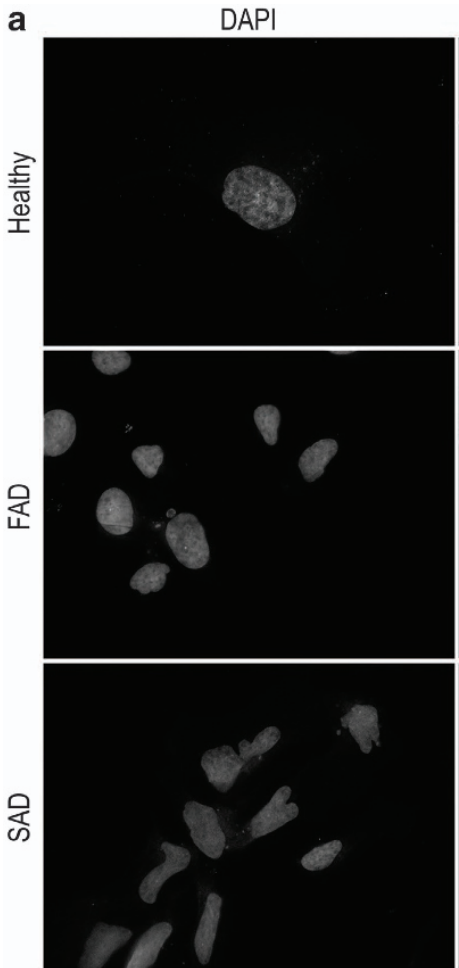

b
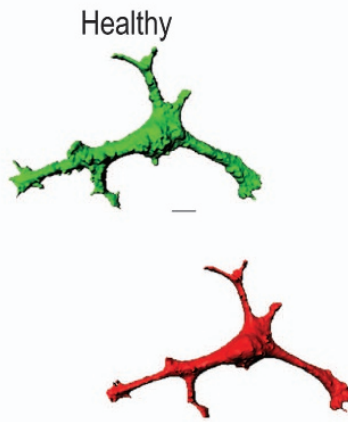

S100B
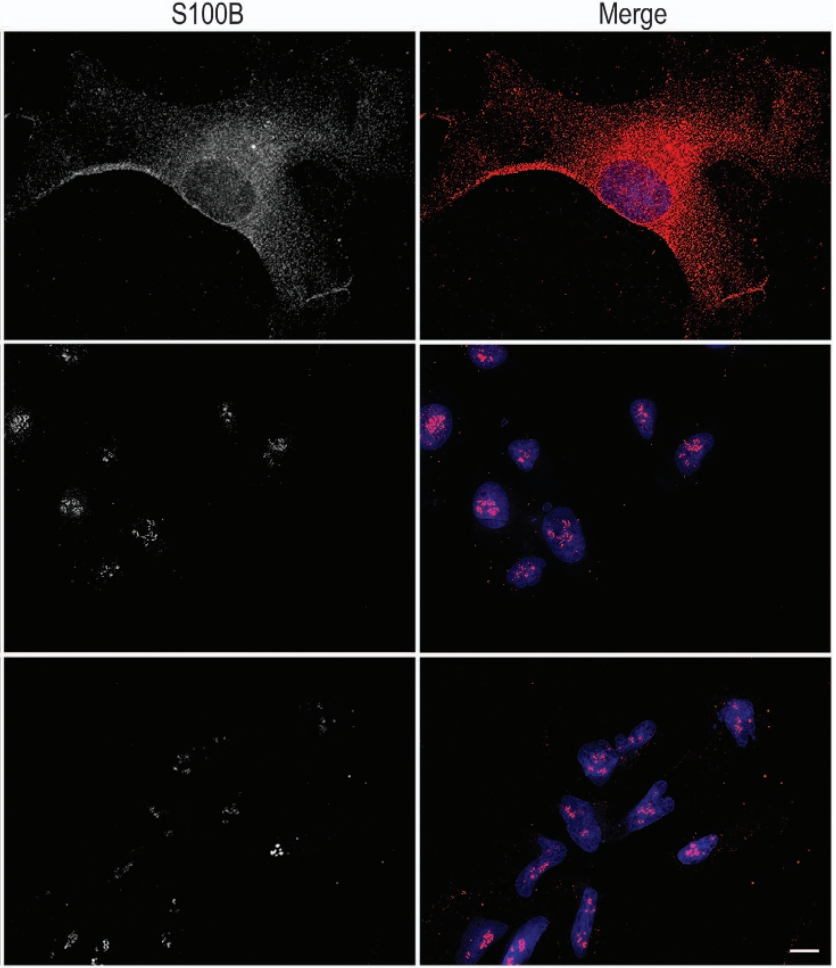

FAD
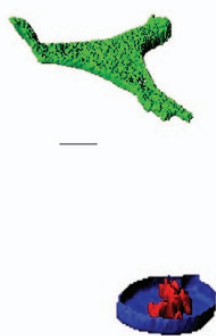

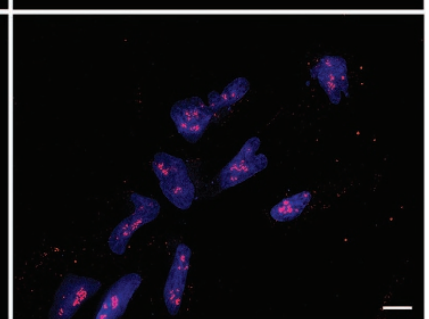

SAD

Figure 6 S100B localisation within astrocytes is dramatically altered in FAD and SAD Healthy astrocytes were immunostained for S100B and visualised using deconvolution fluorescence microscopy (a) revealing S100B to be distributed throughout the cytoplasm (top panel). In contrast, in FAD and SAD cells, S100B appeared to be localised exclusively at the nucleus (middle and bottom panels, respectively). Scale bar, $20 \mu \mathrm{m}$. IsoSurface renders of S100B (red) and of DAPI-stained nuclei (blue; with a clipping plane set to permit visualisation of the inside of the nucleus) were constructed from serial confocal z-sections to further investigate the subcellular localisation of S100B (b; scale bars, $10 \mu \mathrm{m})$. GFAP staining (green) is shown for comparison for healthy cells to reveal the overall cell morphology. In healthy cells, as expected, S100B displayed a cytosolic distribution, similar to that of GFAP. In both FAD and SAD astrocytes, S100B is localised exclusively to discrete foci within the nucleus. Nuclei images have been zoomed in $\times 2$ for clarity. Movies of these renders can be viewed in Supplementary Movies S1-S3

in vitro and are partially compromised in both FAD and SAD astrocytes.

\section{Discussion}

Since astrocytes are the main homeostatic units of the CNS, their dysfunction may drive progression of neurologic disorders, including AD. Astrocytes contribute to non-cell autonomous mechanisms in various neurodegenerative disorders that were previously thought of as classically neuronal diseases. ${ }^{28,29}$ Here we report novel pathological astroglial phenotypes in both FAD and SAD through exploitation of an iPSC-based human model. We show evidence to support critical astroglial contribution to AD namely: (i) mislocalisation and abnormal expression of mature astrocyte markers, (ii) compromised astrocyte heterogeneity and (iii) astroglial atrophy. Singularly, we did not observe differences in early maturation of $\mathrm{AD}$-derived neurones compared to healthy controls, showing that astroglial changes are cell autonomous and do not derive from compromised neuronal intermediates (Figure 7). Generation of functional astrocytes from healthy iPSCs has previously been reported to be time-consuming and result in 20-30\% contamination of unwanted cell types. ${ }^{18-20}$ In contrast, we demonstrate highly efficient generation of enriched populations of mature cortical astrocytes (>95\%), irrespective of donor origin, within 30 days of 

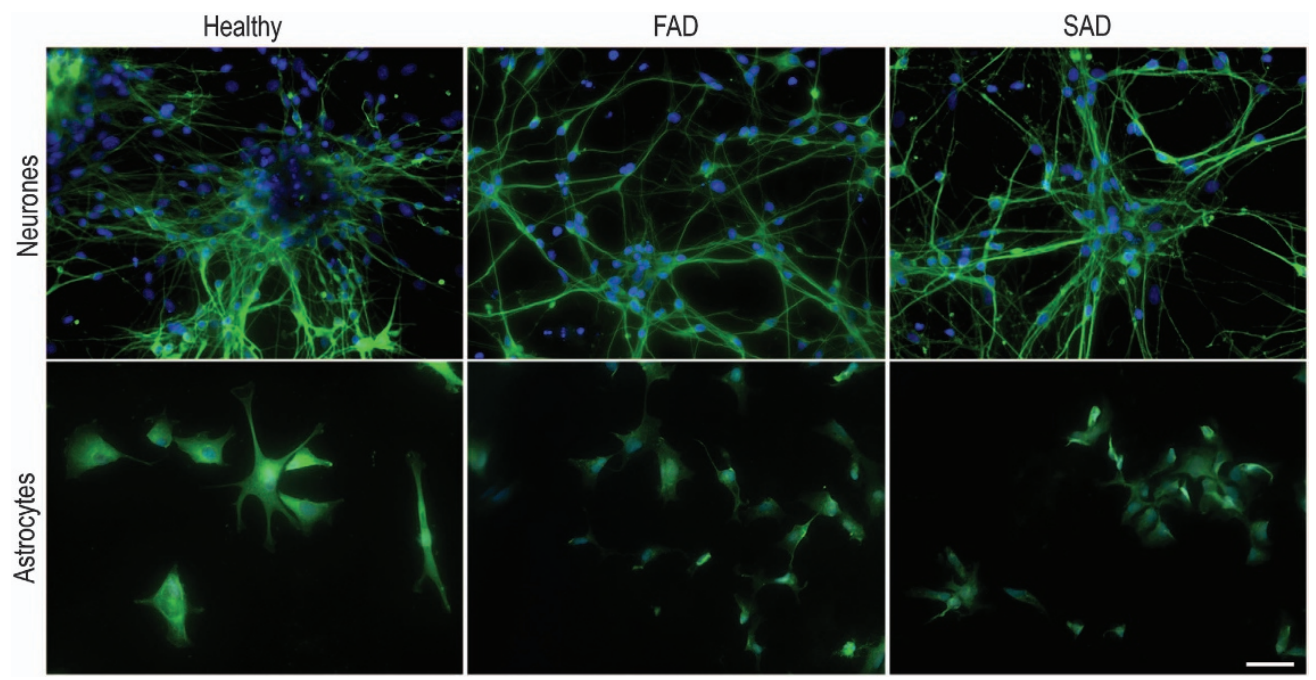

Figure 7 Comparison of healthy control, FAD and SAD patient-derived $\beta$ III-tubulin immunoreactive neurones and GFAP immunoreactive astrocytes. Early neuronal appearance is indistinguishable across the groups, whereas $A D$ astrocytes show markedly reduced heterogeneity of morphology and striking atrophy compared with healthy cells. Scale bar, $50 \mu \mathrm{m}$

induction. This is likely due to additional medium supplementation of EGF and insulin, both of which have been previously shown to increase glial commitment and primary astrocyte proliferation in vitro. ${ }^{26,27}$ Owing to a lack of astrocyte markers that allow precise examination of subtypes that occur throughout the CNS, we were not able to confirm their cortical identity. However, it has been previously published that maturation of neurones or glia from regionally specified iPSC-derived NPCs maintain their identity. ${ }^{30}$ Critically, the healthy iPSC-derived astrocytes described herein were comparable in their morphology and marker expression to those described in several previously published independent studies; validating our differentiation protocol and model. ${ }^{18,19}$

Astrocytes exhibited 95-98\% expression of GFAP, EAAT1, GS and S100B irrespective of healthy or disease origin. However, we observed significant redistribution and decreased expression of S100B in FAD and SAD astrocytes compared with healthy cultures. Although nuclear S100B expression has previously been reported, ${ }^{18-20}$ its expression has not been shown to be exclusively restricted to the nucleus/ nucleoli. Why S100B would localise to a nuclear subcompartment in pathologically remodelled astrocytes remains to be established, but given that $\mathrm{S100B}$ is known to interact with various cytoskeletal components, together with the reduction in its expression levels, may represent a novel and early mechanism underlying SAD- and FAD-induced astrocytic atrophy. Mislocalisation of astrocytic GS was also observed in FAD and SAD astrocytes, however, altered subcellular EAAT1 distribution was only observed in cultures derived from SAD cells. Previous studies have shown altered glutamatergic signalling in neuronal $A D$ degeneration. ${ }^{31-33}$ Our results further reveal a previously unrecognised astroglial cell autonomous pathological phenotype in $A D$, although a significant reduction in the overall fluorescent intensities of EAAT1 and GS was only seen in FAD-derived astrocytes. This finding echoes our recent report of a significant decrease in GS expression in the frontal cortex of 3xTg-AD murine model, ${ }^{34}$ and may hint that astroglia-associated glutamate turnover differs between the two forms of $A D$ at an early stage.

We found that healthy induced astrocytes show significant morphological heterogeneity, in accordance with morphologically distinct cortical astrocytic subpopulations in vivo, ${ }^{35}$ further validating the use of our cell-based model for the interrogation of astroglial function. In contrast, FAD and SAD astrocytes have reduced heterogeneity, are significantly smaller than their healthy counterparts and exhibit an almost complete absence of processes. This corroborates recent studies in mouse models of FAD showing reduced somata and process volumes in astrocytes together with a reduction in the number and complexity of astrocyte processes are an early feature of the disease..$^{10,11,36,37}$ Our results confirm that cell autonomous astrocytic atrophy is a feature of early-onset FAD, but also provide evidence that astrocytic aberrance is a likely characteristic of SAD.

We also show evidence for an altered basal astrocyte secretome profile in both FAD- and SAD- derived cells. Our study of non-stimulated healthy astrocytes in culture express a similar array of inflammatory mediators previously identified in cultures from highly purified human fetal and adult astrocytes. ${ }^{38,39}$ Furthermore, these mediators have been previously shown to be direct targets of the transcription factor NF- $k$ B indicating that this pathway may be constitutively active in human astrocytes under two-dimensional culture conditions. ${ }^{38,39}$ We also observed significantly compromised constitutive secretion of the pro-inflammatory mediators; IL-8 (CXCL8) and MCP-1 (CCL2) in FAD- and SAD-derived ACM, both of which have been previously shown to be the most abundantly expressed transcripts in adult healthy human astrocytes in vitro. ${ }^{39}$ These early observations may indicate a potential glial paralysis as such, which has been postulated to be a fundamental factor in the evolution of AD. ${ }^{14}$ Although, herein we describe the use of a single patient-derived cell line for each criteria, it is reasonably common-place that articles in this field are published whereby studies describe only a single 
patient-derived cell line due to the labour-intensive and costprohibitive nature of induced pluripotent stem cell-derived culture methods and differentiation protocols. ${ }^{17,40-45}$

However, it should be emphasised that cultured astrocytes utilised in the present study represent a simplified model relative to that of astrocytes in the CNS, whereby additional interactions with other cell types and matrix components are likely to influence the astrocytic phenotype. Yet, our highly purified human astrocyte culture model presents a unique system to delineate the autonomic responses of astrocytes to defined stimuli/matrix/co-cultures in both healthy and ADaffected cells.

\section{Conclusions}

Using a human in vitro iPSC model of familial and sporadic $A D$, we demonstrate a significant and previously unknown cell autonomous pathological phenotype of astroglia. Crucially, neuronal intermediates derived from identical iPSCs obtained from the same patients, did not show any pathological phenotype when compared with healthy controls, further corroborating recent ideas of the fundamental role of astroglia in the development of neurodegenerative diseases. We may further conjecture that early synaptic dysfunction, arising from the inhibition of astrocyte-synapse interplay and disruption of functional astroglial networks, is an early feature of $A D$ progression, supporting the idea of astrocytic atrophy as a plausible mechanism for early cognitive impairment and thus providing a potential novel therapeutic target for $A D$ intervention.

\section{Materials and Methods}

All reagents were purchased from ThermoFisher (Paisley, UK) unless stated otherwise.

Derivation and maintenance of neuronal cell lineages. Human iPSC-derived cortical neural progenitor cells (NPCs) were supplied by Axol Bioscience (Cambridge, UK) and generated from patient dermal fibroblasts exhibiting an M146L mutation in the presenellin-1 gene (PSEN1) from a 53-year-old male donor clinically affected with type III early-onset FAD and from an 87-year-old female clinically affected with late-onset SAD homozygous for the four allele of apolipoprotein $\mathrm{E}\left(\mathrm{ApoE}^{+/+}\right)$. The control samples were supplied by Axol Bioscience and reprogrammed from human female umbilical cord blood cells using the same method. NPCs were expanded following manufacturer guidelines and expression of known NPC markers were confirmed before astroglial differentiation.

For directed astroglial differentiation, NPCs were dissociated using accutase and attached with a substrate of poly-L-ornithine $(20 \mu \mathrm{g} / \mathrm{ml}$; Sigma, Dorset, UK) and mouse laminin $(10 \mu \mathrm{g} / \mathrm{ml})$ in chemically defined medium modified from Shaltouki et al. ${ }^{18}$ comprising neurobasal medium containing B27 supplement $(1 \mathrm{x})$, nonessential amino acids $(1 \times)$, L-glutamine $(2 \mathrm{mM})$, penicillin/streptomycin $(50 \mathrm{U} / \mathrm{ml})$, $8 \mathrm{ng} / \mathrm{ml} \mathrm{FGF2} \mathrm{(Peprotech,} \mathrm{London,} \mathrm{UK),} 5 \mathrm{ng} / \mathrm{ml} \mathrm{CNTF}$ and $10 \mathrm{mg} / \mathrm{ml} \mathrm{BMP2}$ (Peprotech) with additional supplementation of $10 \mathrm{ng} / \mathrm{ml} \mathrm{EGF}$ and $5 \mu \mathrm{g} / \mathrm{ml}$ insulin. NPCs were seeded at $3 \times 10^{4} \mathrm{celll} / \mathrm{cm}^{2}$ and maintained at $37^{\circ} \mathrm{C} / 5 \% \mathrm{CO}_{2}$. The cells were cultured until $80 \%$ confluence (approximately 5 days) and passaged twice, as previously detailed. On the third passage, and all consecutive passages, the cells were plated onto non-coated tissue culture plastic to remove unwanted neurones and enrich for astroglia due to their superior adhesiveness. The cells were passaged at least three times during astroglial commitment. Astrocytes were generated a minimum of three times using independent differentiations from each patient sample.

For terminal neuronal differentiation, NPCs were seeded as above, but onto plates coated with $20 \mu \mathrm{g} / \mathrm{ml}$ poly-L-ornithine and $10 \mu \mathrm{g} / \mathrm{ml}$ laminin. The cells were maintained in a 1:1 mixture of N2:B27 media; where N2 medium comprised;
DMEM/F-12 with Glutamax, N2 supplement $(1 \times), 1 \mathrm{mM}$ L-glutamine, non-essential amino acids $(1 \times), 0.1 \mathrm{mM} \beta$-mercaptoethanol, $50 \mathrm{U} / \mathrm{ml}$ penicillin/streptomycin and $5 \mu \mathrm{g} / \mathrm{ml}$ insulin (Sigma); and B27 medium comprised Neurobasal, B27 supplement $(1 \times), 200 \mathrm{mM}$ L-glutamine, $50 \mathrm{U} / \mathrm{ml}$ penicillin/streptomycin. Terminally differentiated neurones were maintained for 30-40 days with medium changes every other day. The cell counts and viability were performed using Trypan blue exclusion assay and calculated using an automated cell counter. Population doubling times were calculated when viable cells exhibited exponential growth. Neurones were generated a minimum of three times using independent differentiations from each patient sample.

Immunocytochemistry. The cells were fixed in $4 \%(\mathrm{w} / \mathrm{v})$ paraformaldehyde in phosphate-buffered saline (PBS) for $15 \mathrm{~min}$ and then treated with $0.1 \mathrm{M}$ glycine in PBS for a further $10 \mathrm{~min}$ to quench unreacted aldehydes. Fixed cells were permeabilised by incubation in $0.1 \%(\mathrm{w} / \mathrm{v})$ Triton $\mathrm{X}-100$ in PBS for $5 \mathrm{~min}$ and blocking of nonspecific binding was achieved by incubation with $1 \%(\mathrm{w} / \mathrm{v})$ bovine serum albumin (BSA) in PBS for $1 \mathrm{~h}$. The cells were subsequently incubated for $30 \mathrm{~min}$ in $1 \% \mathrm{BSA} / \mathrm{PBS}$ containing rabbit polyclonal IgG antiserum for the glial fibrillary acidic protein (GFAP; 1:100; Sigma), mouse monoclonal clone $1 \mathrm{~B} 2 \lg \mathrm{g} 1 \kappa$ for S100 calcium-binding protein B (S100B; 1:100; Sigma), rabbit polyclonal IgG antiserum for the excitatory amino acid transporter-1, (EAAT1; 1:200; Abcam, Cambridge, UK), goat polyclonal antiserum for glutamine synthetase (GS; C-20; 1:100; Santa Cruz Biotechnology, Dallas, TX, USA), rabbit polyclonal IgG antiserum paired box-6 (PAX6; 1:100; BioLegend, London, UK), recombinant monoclonal rabbit IgG sex determining region-1 (SOX1; 1:100; Abcam), monoclonal mouse IgG1 nestin (1:200; Abcam) or COUP-TF-Interacting Protein 2 (CTIP2; 1:300; Abcam). Appropriate Alexa 488 or Alexa 546-conjugated fluorescent secondary antibodies in $1 \%$ BSA/PBS with $0.1 \mu \mathrm{g} / \mathrm{ml} \mathrm{DAPI}$ were applied (Sigma) for a further 30 min. Coverslips were mounted with ProLong Diamond antifade mountant, cured overnight and sealed with nail varnish before imaging.

Fluorescence microscopy and image analysis. The fixed cells were visualised using a Zeiss Cell Observer Z-sectioning fluorescence imaging system equipped Zeiss definite focus, HXP $120 \mathrm{~V}$ illumination and FITC, DsRed and DAPI filter sets. Fluorescence images were acquired using $\times 20 \mathrm{PL}$ Apo $(0.8 \mathrm{NA}), \times 40$ EC Plan-Neofluar (1.3 NA) oil and $\times 63$ PL Apo (1.4 NA) oil objectives, AxioCam MRm Rev. 3 CCD camera and ZEN Pro software (Carl Zeiss, Cambridge, UK). The images were collected as serial optical z-sections taken at $0.28 \mu \mathrm{m}$ intervals, which were subsequently deconvolved using a fast iterative algorithm with a maximum of five iterations. The $z$-stacks were processed and analysed using FIJI ImageJ. ${ }^{46,47}$ All the images presented herein are maximum intensity projections of these z-stacks unless otherwise indicated. Morphological analysis was carried out by visually binning cells into one of three categories: fibroblast-like process-devoid cells, process bearing arborised cells and polarised cells. A minimum of 130 cells drawn from three separate batches of differentiated cells were analysed at $\times 20$ magnification. Immunofluorescence intensity analysis was performed on a minimum of five random fields for each of three separate batches of differentiated cells of view at $\times 20$ magnification using Fiji ImageJ. Z-stacks were flattened before the average background fluorescence, calculated from three random regions of each field of view, was subtracted. Raw integrated pixel densities of each image were calculated and divided by the number of immunoreactive cells to determine an average integrated pixel density per cell for each group.

To permit quantification of GFAP cytoskeleton volume and surface area, serial optical z-sections were collected at $0.5 \mu \mathrm{m}$ intervals using a Leica TCS SP5 AOBS confocal microscope equipped with a $\times 63 \mathrm{HCX}$ PL Apo (0.60-1.40 NA) oil objective, $405 \mathrm{~nm}$ and $488 \mathrm{~nm}$ laser lines and internal PMTs (Leica Microsystems (UK) Ltd, Milton Keynes, UK). Throughout all experiments, the pinhole was set to one Airy unit. The images were collected sequentially to minimise crosstalk using a bidirectional scan at $1000 \mathrm{~Hz}$ and three frame averages. Z-stacks were carefully created so as to include the entire thickness of each cell. Images were subsequently analysed using Imaris 7.7.2 3D visualisation software (Bitplane AG, Zurich, Switzerland) using the IsoSurface tool to create a smoothed 3D surface render of each cell by demarcating the edges of the GFAP-positive cytoskeleton and removing voxels before calculating GFAP surface area, volume and mean fluorescence intensity. A similar approach was used to determine the subcellular localisation of S100B by creating 3D surface renders of both $\mathrm{S100B}$ staining and DAPI-stained nuclei, before using a clipping plane to slice the DAPI channel only, revealing S100B staining within the nucleus. In each experimental group, a minimum of 21 randomly selected cells drawn from three separate differentiations were analysed. To quantify neural progenitor-, neurone- and 
astroglial-marker expression, the cell counts were expressed as a percentage of total cells in a field of view, where the total number of cells was identified using DAPIstained nuclei. The images were captured under standard epifluorescence on an Olympus IX71 microscope (Olympus, Southend-on-Sea, UK) and analysed using ImageJ. Five randomly chosen fields from three independent experiments were assessed.

Antibody arrays. ACM was collected following 30 days in culture and stored at $-80^{\circ} \mathrm{C}$ analysis of inflammatory mediators using a human antibody array kit (Abcam). ACM was collected following cell exposure for $48 \mathrm{~h}$ (normalised to cell number) and samples analysed in parallel to enable comparisons in relative expression levels between experiments. Each experiment represents pooled medium from three independent differentiations. Before analysis, all the samples were thawed on ice and centrifuged at $10000 \times g$ for 5 min at $4^{\circ} \mathrm{C}$. Cytokine/ chemokine secretion was measured according to the manufacturer's instructions. Since, recombinant growth factors are present in ACM, a media-only control was also run in parallel to normalise the data. Positive immunoreactivity was visualised using chemiluminescence using a CCD camera (Bio-Rad, Hertfordshire, UK) and positive/negative membrane controls permitted densitometric analysis using Imagelab software (Bio-Rad).

Statistical analysis. All statistical analyses were carried out using IBM SPSS 22 (IBM Corp., Armonk, NY, USA). Data distributions were analysed by plotting histograms and Quantile-Quantile plots followed by Shapiro-Wilks tests to determine normality. For cell derivation and population analyses, each experiment was performed a minimum of three times and all assays were repeated in triplicate. Mean proportions of nestin+ NPCs and $\beta \| l l-$ tubulin+ neurones were compared by one-way ANOVA. Analyses of cell viability and proliferation rates, cell morphologies and immunofluorescence intensity (with the exception of $\mathrm{S100B}$ ) was carried out using Kruskal-Wallis tests followed by pairwise comparisons by the Dunn-Bonferron method. S100B immunofluorescence intensity comparison was carried out by ANVOA followed by a Hochberg's GT2 post hoc. All the data are presented as mean \pm standard error. An alpha level of 0.05 was considered significant throughout.

\section{Conflict of Interest}

The authors declare no conflict of interest.

Acknowledgements. Special thanks go to Dr. Peter March and Dr. Roger Meadows for their help with the microscopy and to Dr. Christopher Ward for the provision of equipment and facilities in his laboratory. This work was supported by a Seedcorn grant from the University of Manchester Institute for Collaborative Research on Ageing and Dementia@ Manchester fund to LM and VCJ. The University of Manchester Bioimaging Facility confocal microscope and Imaris software used in this study were purchased with grants from BBSRC, Wellcome Trust and the University of Manchester Strategic Fund. Use of the deconvolution imaging system was provided in kind by the University of Central Lancashire.

1. Verkhratsky A, Butt AM. Glial Physiology and Pathophysiology. Wiley-Blackwell: Chichester, UK, 2013.

2. Zhou Y, Danbolt NC. GABA and Glutamate Transporters in Brain. Front Endocrinol 2013; 4 : 165.

3. Burda JE, Bernstein AM, Sofroniew MV. Astrocyte roles in traumatic brain injury. Exp Neurol 2016; 275(Pt 3): 305-315.

4. Sofroniew MV. Astrogliosis. Cold Spring Harb Perspect Biol 2015; 7: a020420.

5. Pekny M, Pekna M, Messing A, Steinhauser C, Lee JM, Parpura V et al. Astrocytes: a central element in neurological diseases. Acta Neuropathol 2016; 131: 323-345.

6. Verkhratsky A, Sofroniew MV, Messing A, deLanerolle NC, Rempe D, Rodriguez JJ et al. Neurological diseases as primary gliopathies: a reassessment of neurocentrism. ASN Neuro 2012; 4: e00082.

7. Chung WS, Welsh CA, Barres BA, Stevens B. Do glia drive synaptic and cognitive impairment in disease? Nat Neurosci 2015; 18: 1539-1545.

8. Molofsky AV, Krencik R, Ullian EM, Tsai HH, Deneen B, Richardson WD et al. Astrocytes and disease: a neurodevelopmental perspective. Genes Dev 2012; 26: 891-907.

9. Verkhratsky A, Olabarria M, Noristani HN, Yeh CY, Rodriguez JJ. Astrocytes in Alzheimer's disease. Neurotherapeutics 2010; 7: 399-412.

10. Olabarria M, Noristani HN, Verkhratsky A, Rodriguez JJ. Concomitant astroglial atrophy and astrogliosis in a triple transgenic animal model of Alzheimer's disease. Glia 2010; 58 : 831-838.
11. Beauquis J, Pavia P, Pomilio C, Vinuesa A, Podlutskaya N, Galvan V et al. Environmental enrichment prevents astroglial pathological changes in the hippocampus of APP transgenic mice, model of Alzheimer's disease. Exp Neurol 2013; 239: 28-37.

12. Beauquis J, Vinuesa A, Pomilio C, Pavia P, Galvan V, Saravia F. Neuronal and glial alterations, increased anxiety, and cognitive impairment before hippocampal amyloid deposition in PDAPP mice, model of Alzheimer's disease. Hippocampus 2014; 24: 257-269.

13. Rodriguez-Vieitez E, Leuzy A, Chiotis K, Saint-Aubert L, Wall A, Nordberg A. Comparability of [18 F]THK5317 and [11C]PIB blood flow proxy images with [18 F]FDG positron emission tomography in Alzheimer's disease. J Cereb Blood Flow Metab 2016; 37: 740-749.

14. Verkhratsky A, Marutle A, Rodríguez-Arellano JJ, Nordberg A. Glial asthenia and functional paralysis. A new perspective on neurodegeneration and Alzheimer's disease. Neuroscientist 2015; 21: 552-568.

15. Gotz J, Ittner LM. Animal models of Alzheimer's disease and frontotemporal dementia. Nat Rev Neurosci 2008; 9: 532-544.

16. Oberheim NA, Takano T, Han X, He W, Lin JH, Wang F et al. Uniquely hominid features of adult human astrocytes. The J Neuroscience 2009; 29: 3276-3287.

17. Zhang PW, Haidet-Phillips AM, Pham JT, Lee Y, Huo Y, Tienari PJ et al. Generation of GFAP::GFP astrocyte reporter lines from human adult fibroblast-derived iPS cells using zinc-finger nuclease technology. Glia 2016; 64: 63-75.

18. Shaltouki A, Peng J, Liu Q, Rao MS, Zeng X. Efficient generation of astrocytes from human pluripotent stem cells in defined conditions. Stem Cells 2013; 31: 941-952.

19. Krencik R, Zhang SC. Directed differentiation of functional astroglial subtypes from human pluripotent stem cells. Nat Protoc 2011; 6: 1710-1717.

20. Roybon L, Lamas NJ, Garcia-Diaz A, Yang EJ, Sattler R, Jackson-Lewis V et al. Human stem cell-derived spinal cord astrocytes with defined mature or reactive phenotypes. Cell Rep 2013; 4: 1035-1048.

21. Kondo T, Asai M, Tsukita K, Kutoku Y, Ohsawa Y, Sunada Y et al. Modeling Alzheimer's disease with iPSCs reveals stress phenotypes associated with intracellular Abeta and differential drug responsiveness. Cell Stem Cell 2013; 12: 487-496.

22. Liao MC, Muratore CR, Gierahn TM, Sullivan SE, Srikanth P, De Jager PL et al. Single-cell detection of secreted Abeta and SAPPalpha from human IPSC-derived neurons and astrocytes. J Neurosci 2016; 36: 1730-1746.

23. Israel MA, Yuan SH, Bardy C, Reyna SM, Mu Y, Herrera C et al. Probing sporadic and familial Alzheimer's disease using induced pluripotent stem cells. Nature 2012; 482: 216-220.

24. Shi $Y$, Kirwan P, Livesey FJ. Directed differentiation of human pluripotent stem cells to cerebral cortex neurons and neural networks. Nat Protoc 2012; 7: 1836-1846.

25. Mohamet L, Miazga NJ, Ward CM. Familial Alzheimer's disease modelling using induced pluripotent stem cell technology. World J Stem Cells 2014; 6: 239-247.

26. Michler-Stuke A, Wolff JR, Bottenstein JE. Factors influencing astrocyte growth and development in defined media. Int J Dev Neurosci 1984; 2: 575-584.

27. Sanalkumar R, Vidyanand S, Lalitha Indulekha $C$, James J. Neuronal vs. glial fate of embryonic stem cell-derived neural progenitors (ES-NPS) is determined by FGF2/EGF during proliferation. J Mol Neurosci 2010; 42: 17-27.

28. Bradford J, Shin JY, Roberts M, Wang CE, Sheng G, Li S et al. Mutant huntingtin in glial cells exacerbates neurological symptoms of Huntington disease mice. J Biol Chem 2010; 285: 10653-10661.

29. Chen PC, Vargas MR, Pani AK, Smeyne RJ, Johnson DA, Kan YW et al. Nrf2-mediated neuroprotection in the MPTP mouse model of Parkinson's disease: critical role for the astrocyte. Proc Natl Acad Sci USA 2009; 106: 2933-2938.

30. Liu H, Zhang SC. Specification of neuronal and glial subtypes from human pluripotent stem cells. Cell Mol Life Sci 2011; 68: 3995-4008.

31. Miguel-Hidalgo JJ, Wei J, Andrew M, Overholser JC, Jurjus G, Stockmeier CA et al. Glia pathology in the prefrontal cortex in alcohol dependence with and without depressive symptoms. Biol Psychiatry 2002; 52: 1121-1133.

32. Burbaeva G, Boksha IS, Tereshkina EB, Savushkina OK, Starodubtseva LI, Turishcheva MS. Glutamate metabolizing enzymes in prefrontal cortex of Alzheimer's disease patients. Neurochem Res 2005; 30: 1443-1451.

33. Revett TJ, Baker GB, Jhamandas J, Kar S. Glutamate system, amyloid $\beta$ peptides and tau protein: functional interrelationships and relevance to Alzheimer disease pathology. J Psychiatry Neurosci 2013; 38: 6-23.

34. Kulijewicz-Nawrot M, Sykova E, Chvatal A, Verkhratsky A, Rodriguez JJ. Astrocytes and glutamate homoeostasis in Alzheimer's disease: a decrease in glutamine synthetase, but not in glutamate transporter-1, in the prefrontal cortex. ASN Neuro 2013; 5 : 273-282.

35. Emsley JG, Macklis JD. Astroglial heterogeneity closely reflects the neuronal-defined anatomy of the adult murine CNS. Neuron Glia Biol 2006; 2: 175-186.

36. Kulijewicz-Nawrot M, Verkhratsky A, Chvatal A, Sykova E, Rodriguez JJ. Astrocytic cytoskeletal atrophy in the medial prefrontal cortex of a triple transgenic mouse model of Alzheimer's disease. J Anatomy 2012; 221: 252-262.

37. Yeh CY, Vadhwana B, Verkhratsky A, Rodriguez JJ. Early astrocytic atrophy in the entorhinal cortex of a triple transgenic animal model of Alzheimer's disease. ASN Neuro 2011; 3 : 271-279.

38. Choi SS, Lee HJ, Lim I, Satoh J, Kim SU. Human astrocytes: secretome profiles of cytokines and chemokines. PLOS ONE 2014; 9: e92325. 
39. Meeuwsen S, Persoon-Deen C, Bsibsi M, Ravid R, van Noort JM. Cytokine, chemokine and growth factor gene profiling of cultured human astrocytes after exposure to proinflammatory stimuli. Glia 2003; 43: 243-253.

40. Andoh-Noda T, Akamatsu W, Miyake K, Matsumoto T, Yamaguchi R, Sanosaka T et al. Differentiation of multipotent neural stem cells derived from Rett syndrome patients is biased toward the astrocytic lineage. Mol Brain 2015; 8: 31.

41. Chang CY, Chen SM, Lu HE, Lai SM, Lai PS, Shen PW et al. N-butylidenephthalide attenuates Alzheimer's disease-like cytopathy in Down syndrome induced pluripotent stem cell-derived neurons. Sci Rep 2015; 5: 8744

42. Hossini AM, Megges M, Prigione A, Lichtner B, Toliat MR, Wruck W et al. Induced pluripotent stem cell-derived neuronal cells from a sporadic Alzheimer's disease donor as a model for investigating AD-associated gene regulatory networks. BMC Genomics 2015; 16: 84

43. Li T, Pires C, Nielsen TT, Waldemar G, Hjermind LE, Nielsen JE et al. Generation of induced pluripotent stem cells (iPSCs) from an Alzheimer's disease patient carrying a M146I mutation in PSEN1. Stem Cell Res 2016; 16: 334-337.

44. Schroter F, Sleegers K, Van Cauwenberghe C, Bohndorf M, Wruck W, Van Broeckhoven C et al. Lymphoblast-derived integration-free iPSC lines from a female and male Alzheimer's disease patient expressing different copy numbers of a coding CNV in the Alzheimer risk gene CR1. Stem Cell Res 2016; 17: 560-563.

45. Zhang D, Pekkanen-Mattila M, Shahsavani M, Falk A, Teixeira Al, Herland A. A 3D Alzheimer's disease culture model and the induction of P21-activated kinase mediated sensing in iPSC derived neurons. Biomaterials 2014; 35: 1420-1428.
46. Schindelin J, Arganda-Carreras I, Frise E, Kaynig V, Longair M, Pietzsch $T$ et al. Fiji: an open-source platform for biological-image analysis. Nat Methods 2012; 9 : 676-682.

47. Schindelin J, Rueden CT, Hiner MC, Eliceiri KW. The ImageJ ecosystem: an open platform for biomedical image analysis. Mol Rep Dev 2015; 82: 518-529.

(1) Cell Death and Disease is an open-access journal published by Nature Publishing Group. This work is licensed under a Creative Commons Attribution 4.0 International License. The images or other third party material in this article are included in the article's Creative Commons license, unless indicated otherwise in the credit line; if the material is not included under the Creative Commons license, users will need to obtain permission from the license holder to reproduce the material. To view a copy of this license, visit http://creativecommons.org/licenses/by/4.0/

(C) The Author(s) 2017

Supplementary Information accompanies this paper on Cell Death and Disease website (http://www.nature.com/cddis) 